B. Zwissler

\section{Pharmacological treatment of acute respiratory failure}

Summary In the past two decades a variety of pharmacological concepts have been suggested to prevent or treat acute respiratory distress syndrome (ARDS). Compounds discussed in this article include antiphlogistics, mucolytics, antioxidants, $\beta_{2}$-mimetics, antibiotics (SDD) and specific enteral nutrition formulas. Analysis of available data shows that very often - promising results have

Eingegangen: 7. Dezember 2000

Akzeptiert: 10. Februar 2001

Serie:

Die Intensivtherapie

bei akutem Lungenversagen

Herausgegeben von R. Kuhlen und R. Rossaint, Aachen

Bernhard Zwissler (

Klinik für Anästhesiologie

Klinikum der Universität München

Marchioninistr. 15

81377 München

e-mail: Bernhard.Zwissler@ana.med.unimuenchen.de

\title{
Pharmakologische Therapie des akuten Lungenversagens
}

been obtained in initial animal experiments and in small clinical studies, but have not been reproduced in large prospective clinical trials. Although the expected biological drug effects were present in most human studies (e.g., increase of antioxidant capacity), only lowdose methlyprednisolone in late ARDS and enteral nutrition with a diet rich in eicosapentaenoic and -linolenic acid increased the number of ventilator-free days or reduced ICU-stay in ARDS patients. Improved survival has not been shown with any of the pharmacological concepts investigated up to now.

Key words ARDS pharmacological treatment nutrition - glucocorticoids $\beta_{2}$-mimetics - mucolytics antioxidants

Zusammenfassung In den zurückliegenden zwei Jahrzehnten wurden eine Vielzahl pharmakologischer Konzepte hinsichtlich ihrer Effektivität bei der Prophylaxe und Therapie des akuten Lungenversagens (ARDS) untersucht. Hierzu zählen der Einsatz von Antiphlogistika, Antioxidantien, Mukolytika, inhalierten $\beta_{2^{-}}$
Mimetika und Antibiotika ebenso wie die Verabreichung spezifischer Ernährungsdiäten. Die Analyse der vorliegende Daten zeigt, dass sich vielversprechende Ergebnisse aus initialen tierexperimentellen Untersuchungenen oder kleineren klinischen Studien nur selten in großen kontrollierten Studien reproduzieren ließen. Zwar fanden sich auch beim Menschen regelmäßig signifikante biologische Effekte nach Applikation der verschiedenen Pharmaka (z.B. Erhöhung der antioxidativen Kapazität). Eine Reduktion der Beatmungsdauer bzw. der Aufenthaltsdauer auf Intensivstation konnte jedoch bislang nur für die niedrigdosierte Gabe von Methylprednisolon bei therapierefraktärem ARDS sowie für die Applikation einer spezifischen, mit Eicosapentaensäure und $\gamma$-Linolensäure angereicherten enteralen $\mathrm{Er}$ nährungsdiät gezeigt werden. Eine Verbesserung des Überlebens ist bislang für keines der verfügbaren Therapiekonzepte belegt.

Schlüsselwörter ARDS Pharmakotherapie - Ernährung Glucocorticoide - $\beta_{2}$-Mimetika Mucolytika - Antioxidantien 


\section{Einführung}

Eine kausale Therapie des akuten Lungenversagens (ARDS) existiert bis heute nicht. Die Behandlung stützt sich daher überwiegend auf symptomatische Maßnahmen. Ziel dieses Beitrags ist es, den aktuellen Stellenwert der adjuvanten Pharmakotherapie unter Einsatz von Antiphlogistika, Antioxidantien, $\mathrm{Mu}$ kolytika, inhalierten $\beta_{2}$-Mimetika sowie spezifischer Ernährungsdiäten darzustellen sowie Möglichkeiten der pharmakologischen Prophylaxe (z.B. SDD) zu diskutieren. Die Applikation von inhalierbaren Vasodilatatoren, Surfactant oder Perfluorcarbonen (,Flüssigkeitsventilation') stellt ebenfalls eine spezifische Form der Pharmakotherapie des ARDS dar, wird jedoch im Rahmen der Serie ,Intensivmedizin beim akuten Lungenversagen' in eigenen Beiträgen ausführlich diskutiert.

\section{Prophylaxe}

\section{Selektive Darmdekontamination (SDD)}

Ziel der sog. selektiven Darmdekontamination (SDD) ist es, durch Eliminierung potentiell pathogener Keime im Bereich des oberen Magen-Darm-Traktes die Inzidenz nosokomialer Pneumonien bei kritisch kranken, langzeitbeatmeten Patienten zu reduzieren und dadurch letztlich die Letalität zu senken. Die Effektivität dieses Therapiekonzeptes wird insgesamt in der Literatur widersprüchlich beurteilt (Übersicht bei (5)). Studien zum Stellenwert von SDD bei Patienten mit manifestem ARDS liegen bislang nicht vor. Allerdings konnte in einer randomisierten, doppelblinden, placebo-kontrollierten Untersuchungen an 271 kritisch kranken Patienten mit einer erwarteten Beatmungsdauer von mehr als $48 \mathrm{~h}$ gezeigt werden, dass die topische Applikation von Gentamicin 2\%, Polymyxin E und Amphotericin B in Kombination mit der intravenösen Verabreichung von Ceftriaxon $(2 \mathrm{~g})$ gegenüber Placebo sowohl eine signifikante Reduktion der Inzidenz beatmungsassoziierter Pneumonien $(11,4$ vs. $29,3 \%)$ als auch der Dauer des Aufenthaltes auf der Intensivstation (11 vs. 16,5 Tage) zur Folge hatte (5). Zwar ergaben sich hinsichtlich der Letalität in beiden Kollektiven keine Unterschiede $(38,9$ vs. $47,1 \%$; $p=0,57)$, die Therapiekosten waren jedoch je überlebendem Patienten in der Behandlungsgruppe mit $\$ 11,926$ gegenüber $\$ 16,296$ in der Placebo-Gruppe deutlich geringer. Obwohl es sich in der genannten Untersuchung - zumindest zum Zeitpunkt des Studienbeginns - ausdrücklich nicht um Patienten mit ARDS handelte, lassen die Ergebnisse einen benefiziellen Effekt der SDD auch bei Patienten mit manifestem Lungenversagen vermuten.

\section{Therapie}

\section{Antiinflammatorisch wirksame Pharmaka}

\section{Glucocorticoide}

Wegen ihrer anti-inflammatorischen Wirkung wurden Glucocorticoide lange Zeit zur Therapie des ARDS bzw. bei Sepsis eingesetzt. Bei der wissenschaftlichen Überprüfung dieses Therapiekonzeptes konnte jedoch in zwei kontrollierten Studien aus dem Jahr 1987 kein benefizieller Effekt der hochdosierten Gabe von Methylprednisolon $(30 \mathrm{mg} / \mathrm{kg}$ alle 6 Stunden für $24 \mathrm{~h}$ ) beim frühen ARDS nachgewiesen werden $(1,3)$. In einer Studie ergab sich sogar eine signifikant höhere 14-Tage Letalität bei Patienten mit Sepsis-induziertem ARDS (3). Die hochdosierte Gabe von Glucocorticoiden bei ARDS gilt daher heute - wahrscheinlich wegen einer prolongierten und unspezifischen Suppression der Immunantwort - als obsolet.

Im Gegensatz dazu deuten aktuelle Untersuchungen daraufhin, dass die repetitive, ,niedrigdosierte Applikation von Steroiden in der Spätphase des ARDS (z. B. Methylprednisolon 2-3 mg/kg/Tag) Vorteile aufweisen könnte. Mehrfach wurde in diesem Zusammenhang - kürzlich erstmals auch in einer randomisierten, kontrollierten Studie (10) - über eine beeindruckende klinische Besserung von Gasaustausch und Lungenmechanik sowie hohe Überlebensraten bei sonst therapierefraktären Fällen des ARDS berichtet $(6,9)$. Abbildung 1 zeigt den klinischen Verlauf der von Meduri et al. untersuchten Patienten (10): Im Gegensatz zur Placebo-Gruppe verbesserte sich der ,lung injury score' (LIS) in der Behandlungs-Gruppe über einen Zeitraum von 10 Tagen signifikant. Danach waren $43 \%$ der behandelten, aber keiner der nicht behandelten Patienten extubiert. Die Therapie mit Glukokortikoiden wird auf-

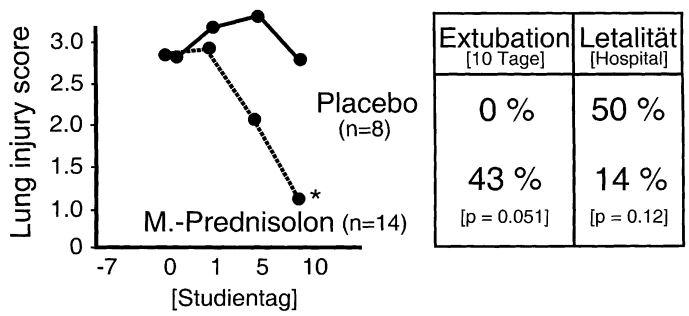

Abb. 1 Methylprednisolon bei therapierefraktärem, spätem ARDS (10). Einfluss auf den ,Lung Injury Score', Extubationsrate und Krankenhausletalität. ${ }^{*} p<0,05$ 
grund dieser vielversprechenden Ergebnisse derzeit von namhaften Autoren als ,Rescue'-Therapie bei ARDS empfohlen (14) und in einer großen multizentrischen Studie (USA) weiter evaluiert.

\section{Andere antiinflammatorisch wirksame Agentien}

Andere, in tierexperimentellen und kleineren klinischen Studien wirksame antiinflammatorische Therapieansätze (Ibuprofen, Ketokonazol, $\mathrm{PGE}_{1}$, Lisophyllin oder Procystein) konnten in großen klinischen Untersuchungen weder die Morbidität noch die Letalität bei ARDS senken (Übersicht bei (14)). Ihr Einsatz bei Patienten mit ARDS ist derzeit nicht indiziert.

\section{Ernährung}

Während die unselektive Hemmung des Cyclooxygenase-Stoffwechsels neben einer Reduktion von Metaboliten mit proinflammatorischer sowie vaso- und bronchokonstriktorischer Wirkung (z.B. LTB $_{4}$, Thromboxan $\mathrm{A}_{2}$ u.a.) gleichermaßen auch eine Hemmung von im ARDS potentiell protektiven Metaboliten (z.B. Prostazyklin) zur Folge hat, scheint der Einsatz spezieller Ernährungsdiäten einen gezielteren Eingriff in die Balance des Arachidonsäurestoffwechsels $\mathrm{zu}$ erlauben. So bewirkte in mehreren experimentellen Studien die Zufuhr von mit $\omega$-3-Fettsäuren angereicherten Diäten (Fischöl) die Synthese von Prostaglandinen und Leukotrienen mit geringerer chemotaktischer, vasokonstriktiver, proinflammatorischer und thrombozyten-aggregierender Wirkung im Vergleich $\mathrm{zu}$ mit herkömmlichen, an $\omega$-6-Fettsäuren-reichen Diät ernährten Tieren. In Übereinstimmung hiermit fand sich in einer experimentellen Studie an Schweinen eine geringere Konzentration an $\mathrm{TxB}_{2}$, eine geringere Zunahme des pulmonalen Gefäßwiderstands bzw. ein geringerer $\mathrm{Ab}$ fall des systemischen $\mathrm{O}_{2}$-Angebots bei denjenigen Tieren, die vor Induktion einer Sepsis (E. coli) über 8 Tage eine an $\omega$-3-Fettsäuren reiche Diät erhielten (12). Diese tierexperimentellen Befunde werden durch eine aktuelle klinische Multizenter-Studie unterstützt (4), in der 146 Patienten mit ARDS randomisiert entweder mit einer enteralen Standarddiät oder aber mit einer an Eicosapentaensäure (Fischöl), $\gamma$-Linolensäure und Antioxidantien (Vit C, E, Carnitin, Taurin) reichen enteralen Diät ernährt wurden. Die so ernährten Patienten wiesen gegenüber der Kontrollgruppe im Verlauf nicht nur eine signifikant bessere Oxygenierung, sondern auch eine niedrigere Inzidenz zusätzlicher Organversagen, eine geringere Anzahl von Beatmungstagen und eine kürzere Behandlungsdauer auf der Intensivstation auf (Abb. 2).

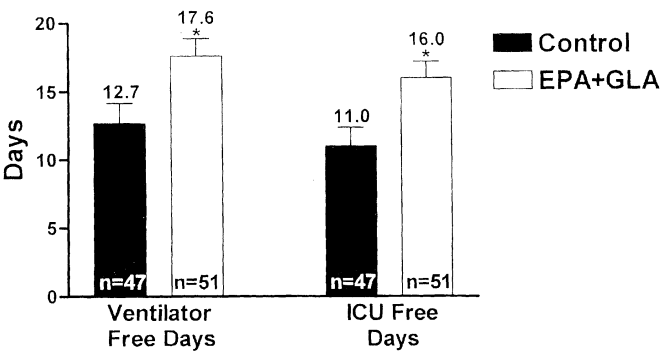

Abb. 2 Einfluss einer modifizierten Ernährungsdiät (EPA +GLA) auf die Dauer der Beatmung und die Aufenthaltsdauer auf der Intensivstation bei ARDS. Schwarze Balken: Standard-Diät; Offene Balken: Testdiät mit Eicosapentaensäure und $\gamma$-Linolensäure

\section{Mukoziliäre Klärfunktion/Sekretolyse/ Antioxidantien}

Beim beatmeten Intensivpatienten kommt es häufig zu einer Störung der mukoziliären Klärfunktion. Die Beeinträchtigung dieses unspezifischen, aber protektiven Schutzmechanismus der Lunge ist mit dem gehäuften Auftreten von Sekretretentionen bzw. von nosokomialen Pneumonien assoziiert. Neben einer Atemgasbefeuchtung, der adäquaten Bronchialtoilette durch wiederholtes (steriles, ggf. bronchoskopisches) Absaugen von Endotrachealsekret sowie physikalischen Maßnahmen zur Schleimlösung (z.B. Vi$\left.\operatorname{brax}^{\circledR}\right)$ hat es nicht an Versuchen gefehlt, die mukoziliäre Klärfunktion und Sekretolyse medikamentös $\mathrm{zu}$ verbessern. So gelang es beispielsweise, durch die Gabe von $1 \mathrm{mg} / \mathrm{kg} / \mathrm{h}$ Theophyllin (Initialdosis $5 \mathrm{mg} /$ $\mathrm{kg}$ ), die Wanderungsgeschwindigkeit von radioaktiv markiertem Albumin auf der bronchialen Schleimhautoberfläche (d.h. die bronchiale Transportgeschwindigkeit) von beatmungspflichtigen Intensivpatienten signifikant zu erhöhen (8). Ähnliche Erfolge wurden jedoch von anderen Untersuchern nicht beobachtet. Darüberhinaus geht die Therapie bei einer Reihe von Patienten mit einer unerwünschten Tachykardie einher. Ihre Anwendung kann daher nicht generell empfohlen werden (8).

Auch die Effektivität der seit langem als Mukolytika bzw. (in höherer Dosierung) als Antioxidantien eingesetzten Substanzen Ambroxol bzw. N-Acetylcystein (ACC) wird in der Literatur kontrovers diskutiert. In einer Untersuchung an beatmeten Patienten erbrachte die Therapie mit ACC über 5 Tage (3 g/Tag) weder hinsichtlich der Zahl der tracheobronchialen Absaugungen, der bronchoskopisch vorgefundenen Sekretmenge oder der Zähigkeit des abgesaugten Sekrets signifikante Unterschiede gegenüber einer unbehandelten Kontrollgruppe (7). Dagegen ließ sich die in zahlreichen tierexperimentellen Untersuchungen beobachtete antioxidative Wirkkomponente von ACC kürzlich erstmals in einer kontrol- 
lierten, multizentrischen Studie bei Patienten mit ARDS reproduzieren (2). So hatte die hochdosierte Applikation von ACC $(70 \mathrm{mg} / \mathrm{kg}$; alle $8 \mathrm{~h}$ für $10 \mathrm{Ta}-$ ge) nicht nur einen signifikanten Wiederanstieg der bei ARDS pathologisch niedrigen Konzentration wichtiger Antioxidantien (Gluthation, Cystein) zur Folge, sondern verminderte auch die Dauer des akuten Lungenversagens. Ein benefizieller Einfluss auf das Überleben der Patienten fand sich allerdings nicht (2). $\mathrm{Zu}$ sehr ähnlichen Ergebnissen kam kürzlich die Arbeitsgruppe um Ortolani et al. (13). Bei 36 Patienten mit frühem ARDS reduzierte ACC in einer Dosierung von $50 \mathrm{mg} / \mathrm{kg}$ die Konzentration von exspiratorischem Ethan, Malondialdehyd und oxidiertem Glutathion, was von den Autoren als lungenprotektiver Effekt interpretiert wurde. Eine Verbesserung der Überlebensrate ließ sich jedoch (u.a. auch wegen der niedrigen Fallzahl) auch in dieser Studie nicht belegen. Ähnlich wie für ACC konnte auch für die hochdosierte Gabe von Ambroxol in einigen klinischen Untersuchungen benefizielle Effekte bei beatmeten Patienten (z.B. Reduktion der Inzidenz von Atelektasen, Scavenging von $\mathrm{O}_{2}$-Radikalen, Prophylaxe des ARDS), bislang jedoch keine Verbesserung des ,Outcome' von Patienten mit ARDS dokumentiert werden.

\section{Inhalierte $\boldsymbol{\beta}_{\mathbf{2}}$-Mimetika}

Die Erkenntnis, dass es bei Patienten mit ARDS neben einer Verminderung der pulmonalen Compliance auch zu einer signifikanten Zunahme des Atemwegswiderstandes durch Tonuserhöhung der glatten Bronchialmuskulatur kommt, ist relativ neu (15) und stellt einen möglichen Ansatz für die pharmakologische Therapie dieses Krankheitsbildes dar. So konnte in zwei klinischen Untersuchungen an insgesamt 19
Patienten mit ARDS gezeigt werden, dass die Verabreichung von inhalierten $\beta_{2}$-Mimetika (z.B. Salbutamol) zu einer signifikanten Abnahme der Atemwegsspitzen- bzw. Plateaudrücke sowie des Atemwegswiderstands bei diesen Patienten führte $(11,15)$. Experimentelle Daten deuten auch auf eine rascheres Verschwinden eines intraalveolären Ödems unter Inhalation von $\beta_{2}$-Mimetika hin (Übersicht bei (14)). Bislang ungeklärt ist jedoch, ob die konsequente pulmonale Bronchodilatation auch mit einer Abnahme des Risikos von Barotraumata einhergeht, wie dies von einigen Autoren vermutet wird.

\section{Zusammenfassung}

In den zurückliegenden zwei Jahrzehnten wurden eine Vielzahl pharmakologischer Konzepte auf ihre Effektivität im Rahmen der Prophylaxe und Therapie des akuten Lungenversagens (ARDS) untersucht. Dabei ließen sich vielversprechende Ergebnisse aus initialen tierexperimentellen Untersuchungen oder kleineren klinischen Studien nur selten in großen kontrollierten Studien reproduzieren. Zwar fanden sich auch beim Menschen regelmäßig signifikante biologische Effekte nach Applikation der verschiedenen Pharmaka (z.B. Erhöhung der antioxidativen Kapazität). Eine Reduktion der Beatmungsdauer bzw. der Aufenthaltsdauer auf Intensivstation konnte jedoch bislang nur für die niedrigdosierte Gabe von Methylprednisolon bei therapierefraktärem ARDS sowie für die Applikation einer spezifischen, mit Eicosapentaensäure und $\gamma$-Linolensäure angereicherten enteralen Ernährungsdiät gezeigt werden $(4,10)$. Eine Verbesserung des Überlebens ist bislang für keines der verfügbaren Therapiekonzepte belegt.

\section{Literatur}

1. Bernard GR, Luce JM, Sprung CL et al (1987) High-dose corticosteroids in patients with the adult respiratory distress syndrome. $\mathrm{N}$ Engl $\mathrm{J}$ Med 317:1565-1570

2. Bernard GR, Wheeler AP, Arons MM et al (1997) A trial of antioxidants Nacetylcysteine and procysteine in ARDS. Chest 112:164-172

3. Bone RC, Fisher CJ Jr., Clemmer TP et al (1987) Early methylprednisolone treatment for septic syndrome and the adult respiratory distress syndrome. Chest 92:1032-1036
4. Gadek JE, Demichele SJ, Karlstad MD et al (1999) Effect of enteral feeding with eicosapentaenoic acid, gamma linolenic acid, and antioxidants in patients with acute respiratory distress syndrome. Crit Care Med 27: 1409-1420

5. Garcia MS, Galache JAC, Diaz JL, Cerda EC, Blasco JR, Aguinaga MAG, Reiz AN, Marin SR, Canaveral JJO, and delCastillo JAS (1998) Effectiveness and cost of selective decontamination in the digestive tract in critically ill intubated patients. Am J Resp Crit Care Med 158:908-916
6. Hooper RG, Kearl RA (1996) Established adult respiratory distress syndrome successfully treated with corticosteroids. South Med J 89:359-364

7. Konrad F, Schoenberg MH, Wiedman $\mathrm{H}$ et al (1995) Die Applikation von N-Acetylcystein als Antioxidans und Mukolytikum beim beatmeten Intensivpatienten. Eine prospektive, randomisierte, placebokontrollierte Doppelblindstudie. Anaesthesist 44:651-658

8. Konrad F, Schreiber T, Hahnel J et al (1994) Einfluss von Theophyllin auf die mukoziliäre Klärfunktion beatmeter Intensivpatienten. Anaesthesist 43:101-106 
9. Meduri GU, Belenchia JM, Estes RJ et al (1991) Fibroproliferative Phase of ARDS - Clinical Findings and Effects of Corticosteroids. Chest 100:943-952

10. Meduri GU, Headley AS, Golden E et al (1998) Effect of prolonged methylprednisolone therapy in unresolving acute respiratory distress syndrome: A randomized controlled trial. J Am Med Assoc 280/2:159-165

11. Morina P, Herrera M, Venegas J et al (1997) Effects of nebulized salbutamol on respiratory mechanics in adult respiratory distress syndrome. Intensive Care Med 23:58-64
12. Murray MJ, Kumar M, Gregory TJ et al (1995) Select dietary fatty acids attenuate cardiopulmonary dysfunction during acute lung injury in pigs. Am J Physiol 269: H2090-H2099

13. Ortolani O, Conti A, De Gaudio AR et al (2000) Protective effects of Nacetylcysteine and rutin on the lipid peroxidation of the lung epithelium during the adult respiratory distress syndrome. Shock 13:14-18
14. Ware L, Matthay MA (2000) The acute respiratory distress syndrome. New Engl J Med 342:1335-1349

15. Wright PE, Carmichael LC, Bernard GR (1994) Effect of bronchodilators on lung mechanics in the acute respiratory distress syndrome (ARDS). Chest 106:1517-1523 